\title{
ГЕРОНТОПСИХИАТРИЯ
}

УДК 616.892.32-053.9:616.071:616-079.2:159.95:159.9.072.7

Для цитирования: Коцюбинская Ю.В., Казаков А.В., Матюшкина А.А., Орлов И.А. Возможности применения нейропсихологических методов исследования при болезни Альцгеймера (на примере клинического случая). Сибирский вестник психиатрии и наркологии. 2021; 2 (111): 64-72. https://doi.org/10.26617/1810-3111-20212(111)-64-72

\section{Возможности применения нейропсихологических методов исследования при болезни Альцгеймера (на примере клинического случая)}

\section{Коцюбинская Ю.В., Казаков А.В., Матюшкина А.А., Орлов И.А.}

ФГБУ «Национальный медицинский исследовательский иуентр психиатрии и неврологии им. В.М. Бехтерева» Минздрава России

Россия, 192019, Санкт-Петербург, ул. Бехтерева, 3

\section{PEЗЮME}

Болезнь Альцгеймера (БА) является самой распространенной причиной когнитивного дефицита в результате утраты синаптических связей у лиц старше 65 лет, достигая в 80\% случаев синдрома деменции. Несмотря на насыщенную и продуктивную историю изучения БА, остается множество пробелов, особенно в плоскости клинических особенностей течения БА. Цель исследования: клинический обзор, проведенный с современных позиций доказательной психиатрической практики, на основе наблюдения прогрессирующего течения когнитивного снижения при болезни БА. Приведен клинический пример нейропсихологического исследования пациентки с вероятностной БА (диагноз по МКБ-10: F00.1 Деменция при БА с поздним началом G30.1+) с интерпретацией полученных данных. Пациентка проходила терапию в «Центре лечения расстройств памяти» ФГБУ «Национальный медицинский исследовательский центр психиатрии и неврологии им. В.М. Бехтерева» МЗ РФ (Санкт-Петербург). Результаты комплексного нейропсихологического обследования пациентки указывают на интеллектуально-мнестическое снижение, зрительно-пространственные нарушения, зрительную предметную агнозию, вторичную акалькулию по префронтальному типу, пространственно-конструктивную апраксию, модально-неспецифические нейродинамические нарушения. Выявлены когнитивные нарушения умеренной степени выраженности. По результатам оценки по трем шкалам получены следующие баллы: краткая шкала оценки психического статуса (MMSE) - 12 баллов, шкала (батарея) лобной дисфункции (FAB) - 11 баллов, госпитальная шкала тревоги и депрессии (HADS) - 13 баллов и 7 баллов. Топический диагноз поражения головного мозга: результаты нейропсихологического обследования указывают на патологическое функционирование лобных (префронтальных и медиобазальных) отделов обоих полушарий головного мозга с вовлечением в патологический процесс теменно-затылочных структур, заинтересованности в процессе гипоталамо-гипофизарных и диэнцефально-стволовых структур головного мозга. Таким образом, нейропсихологическое исследование психических функций пациентов с расстройством памяти с позиции биопсихосоциального подхода детализирует не только когнитивный дефицит, но и степень утраты социальных функций.

Ключевые слова: болезнь Альцгеймера, когнитивные нарушения, умеренный когнитивный дефицит, деменция, нейропсихологические методы, анализ психических функций.

\section{ВВЕДЕНИЕ}

В последние десятилетия перед неврологами и психиатрами во всем мире стоит задача по описанию основных признаков клинической картины, специфических проявлений и симптомов на доклинической стадии и надежности диагностики БА, поиску биохимических, иммунологических, молекулярно-генетических механизмов развития различных форм деменции ввиду важнейшего социального и экономического значения данной социально значимой патологии.
Болезнь Альцгеймера является самой распространенной причиной когнитивного дефицита у лиц старше 65 лет, достигая в $80 \%$ случаев синдрома деменции [1, 2]. В 1907 г. Алоис Альцгеймер (Aloysius “Alöis” Alzheimer) впервые детально описал основные симптомы у находившейся под его наблюдением в городском госпитале Франкфурта 51-летней пациентки Auguste Deter, после смерти которой провел гистологическое исследование её головного мозга путем окрашивания серебром [3]. 
Результатом гистологического исследования стало открытие невральных бляшек, нейрофиблиллярных сплетений и амилоидной ангиопатии, ставших впоследствии опознавательными знаками болезни, в настоящее время носящей его имя [4]. Базируясь на работах Альцгеймера, к 1911 г. медицинское сообщество во всей Европе и США диагностировало данную патологию у пациентов [5]. В 60-х - 70-х гг. прошлого столетия были разработаны стандартизированные тесты для скрининга когнитивных функций, результаты которых высоко коррелировали с БА [6]. В 1976 г. Роберт Катцман (Robert Katzman) провел серию исследований и вывел гистопатологическую идентичность сенильной и пресенильной деменции, а также, опираясь на эпидемиологические данные, заключил, что БА занимает четвертое место среди причин смертности лиц пожилого возраста. Это заключение вывело БА из разряда редко встречающихся заболеваний в одну из главных проблем общественного здравоохранения [7].

Значительным прорывом в оценке клинических проявлений деменции стало осознание того, что сохранность и нарушение когнитивных функций значительно варьируют в зависимости от этиологии патологического процесса. В 1974 г. Мартин Альберт и его коллеги выделили «субкортикальный» и «кортикальный» типы деменции. При обследовании пациентов с прогрессирующим надъядерным параличом были выявлены когнитивные нарушения, характеризующиеся забывчивостью, медлительностью мыслительных процессов, изменениями личности с апатией или депрессией, а также нарушением способности использовать полученные знания. Подобные клинические проявления также были описаны у пациентов с хореей Гентингтона. Такая клиническая картина значительно отличалась от корковой деменции, характерной для БА [8]. Последующие исследования ещё больше подтвердили качественные различия между «подкорковой» и «корковой» деменцией. Итогом стало разделение данных форм деменции на два отчетливых клинических синдрома. В исследованиях 90-х гг. доказано, что амнезия является ранним и наиболее значимым признаком синдрома деменции при БА, что соответствовало патолого-анатомическим исследованиям, показывавшим, что в патологический процесс наиболее вовлечены структуры медиальной височной доли (гиппокамп, энторинальная кора) [9]. Такой дефицит памяти отражал неспособность эффективно обрабатывать и хранить новую информацию, в первую очередь страдало отсроченное вспоминание [9]. Несмотря на такую насыщенную и продуктивную историю изучения, остается множество пробелов, особен- но в плоскости клинических особенностей течения БА.

\section{ЦЕЛЬ ИССЛЕДОВАНИЯ}

Клинический обзор, проведенный с современных позиций доказательной психиатрической практики, на основе наблюдения прогрессирующего течения когнитивного снижения при болезни БА.

\section{МАТЕРИАЛЫ И МЕТОДЫ}

Приведен клинический пример нейропсихологического исследования состояния высших психических функций пациентки в возрасте 66 лет с вероятностной БА (верифицированный по МКБ10 диагноз: F00.1 Деменция при БА с поздним началом G30.1+). Пациентка наблюдалась и обследована в «Центре лечения расстройств памяти» ФГБУ «Национальный медицинский исследовательский центр психиатрии и неврологии им. В.М. Бехтерева» МЗ РФ (Санкт-Петербург).

\section{РЕЗУЛЬТАТЬ}

Сбор жалоб выполнен по данным стандартного опроса сына пациентки. В последнее время стала рассеянной, забывчивой, мало интересуется происходящими событиями, фон настроения снижен, наблюдаются интрасомнические нарушения, снижение массы тела за последние 6 месяцев. Сын отмечает частые случаи рассеянного внимания матери: забывает о намеченных встречах и делах, выражает излишнее беспокойство и избыточное преждевременное волнение задолго до наступления ожидаемого события, утратила стремление к освоению новых видов деятельности, приобретению новых знаний (не хочет читать, отказывается осваивать новые технические средства - телефон, планшет). Критика к собственному состоянию снижена - замечает только грубые виды дезориентировки при выполнении повседневной бытовой деятельности; случаи забывчивости остаются незамеченными. При выполнении повседневных дел, требующих высокой целенаправленности и регуляции поведения, объясняет свою несостоятельность отсутствием мотивации и потребностей.

Из анамнеза жизни известно, что пациентка имеет высшее гуманитарное образование. В настоящий момент находится на пенсии по возрасту. Проживает самостоятельно, однако нуждается в ежедневной социальной поддержке. В ситуации обследования доброжелательна, немногословна.

Проведено исследование состояния отдельных психических функций, позволившее выявить следующие особенности.

Кинестетический мануальный праксис: первичные и вторичные нарушения движений, ригидное использование пальцев, участвовавших в предыдущей пробе, при воспроизведении по- 
следующих поз, зеркальные нарушения при воспроизведении положений пальцев обеих рук (позы 1-4).

Динамический мануальный праксис: вторичные нарушения, выраженные трудности при усвоении трехкомпонентной инструкции, нарушение пространственной организации движений, упрощение программы деятельности.

Конструктивный и пространственный праксис: первичные и вторичные нарушения. Самостоятельное изображение простых геометрических фигур возможно, копирование и дорисовывание простых фигур возможно. Выполнение заданий, требующих высокой степени задействования зрительно-пространственных функций, малодоступно. Самостоятельный рисунок бытовых трехмерных предметов (стол) доступен, низкочастотных предметов (куб) - доступен только по образцу. Расстановка стрелок на «слепых» часах нарушена. Копирование фигур с переворотом только зеркальное.

Соматопространственный праксис: зеркальные ошибки при выполнении проб Хэда и переносе поз с одной руки на другую.

Оральный праксис и состояние артикуляционного аппарата: ограничений объема движений не зафиксировано.

Оральный кинестетический праксис: выполнение простых оральных действий после показа задания специалистом доступно.

Символический оральный праксис: нарушений не выявлено.

Зрительный предметный гнозис: первичные нарушения средней степени, узнавание реальных цветных изображений доступно. Узнавание реалистичных изображений осуществляется со специфическими ошибками по внешне-зрительному сходству. Узнавание перечеркнутых, наложенных и недорисованных изображений доступно со специфическими ошибками интерпретации фона как фигуры.

Зрительный пространственный гнозис: опознание времени на «слепых» часах недоступно, объектная ориентация в схеме географической карты нарушена. Автобиографическая память нарушена, так как успешно воспроизводит лишь факты, соответствующие началу и расцвету своей профессиональной деятельности, состава/членов семьи, однако сбивчиво описывает события последних месяцев, забывает имена, не может вспомнить места, где была с сыном в последнее время. Память на текущие события так же нарушена - дезориентирована в окружающем пространстве и времени. Не следит за режимом дня, что требует внешнего контроля со стороны сына за его соблюдением. Помимо того, пациентка самостоятельно отмечает, что с трудом ориентиру- ется в текущих событиях, в связи с чем думает об использовании записной книжки.

Слухоречевая память: объем непосредственного запоминания существенно снижен и составляет 3 элемента при предъявлении серии из 10 слов. Кривая запоминания: 3/4/5/6/8/8/in ret $3 \mathrm{~min}-9 /$ in ret $40 \mathrm{~min}-7$. Комментировала выполнение задания: «что-то очень много», «это для меня всё очень сложно». После первых двух попыток оценивала общий объём слов как 5-6. После 3-й попытки были предложены ассоциативные подсказки, которые улучшили воспроизведение до 100\%; а также было рекомендовано пересчитать слова на пальцах. После 4-й попытки верно оценила общий объём как 10 слов. При 5-й и 6-й попытках при воспроизведении загибала пальцы по настоянию специалиста, что заметно повысило продуктивность запоминания и помогло при обоих отсроченных воспроизведениях.

Зрительная память: нарушения грубой степени. При первом воспроизведении серии из предъявленных 6 трудновербализуемых фигур изобразила только первую фигуру. Кривая воспроизведения 1//2/2/3/4/4//in ret - 4. Фигуры повторяются с упрощением деталей, последовательность двух последних фигур и полнота изображения первой фигуры флюктуируют от пробы к пробе, что свидетельствует о выраженных флюктуациях внимания и работоспособности при выполнении неречевого когнитивного задания.

Понимание ситуативной речи безошибочное. Понимание внеситутивной речи ограниченное: при выполнении «квазипространственных» инструкций отображает исключительно общий контекст инструкции, однако самостоятельно отмечает, что неточно понимает, что необходимо сделать. Понимание предложенных пассивных логико-грамматических конструкций и конструкций, содержащих временные отношения, осуществляет нерегулярно. Продуктивность понимания инвертированных и сравнительных конструкций несколько снижена. Спонтанная речь представлена фразами, разнообразными по синтаксическому строению и лексическому составу. Вместе с тем подробно отвечает на вопросы. Автоматизированная и дезавтоматизированная речь без нарушений. Договаривание фраз с жестким контекстом безошибочное. Повторение отдельных гласных и согласных звуков, слогов и их серий доступно. Повторение сложных и простых по звукобуквенной структуре слов, в том числе несуществующих, безошибочно. При повторении серий из 3 слов отмечаются перестановки слов. При исследовании номинативной речи вторичные нарушения в связи с трудностями зрительного опознания изображения. При составлении предложе- 
ний трудности при зрительном опознании изображений на картинке.

В случае успешного опознания предметов составление полных, синтаксически правильных фраз доступно. Составление простого связного, грамматически правильного рассказа по сюжетной картинке доступно. Однако затруднено целостное осмысление/понимание сюжетной картинки, что приводит к неправильной оценке роли отдельных персонажей. Чтение простых частотных слов и рассказа доступно. Литеральных паралексий не зафиксировано. Пересказ прочитанного простого текста лаконичное, с аллюзиями и личностными ассоциациями.

Автоматизированное письмо сохранно. Безошибочное написание под диктовку отдельных букв, слогов и простых слов доступно. При написании сложных слов и предложений отмечаются несистемные ошибки: пропуски букв и слогов, персеверации. Счетная деятельность грубо нарушена. Выполнение арифметических действий (процесс счета, итоговое число) заменяется перебором отдельных цифр. Понимание переносного смысла пословиц и метафор нерегулярное, в ряде случаев по ситуативным признакам. Категориальный уровень обобщения при выполнении операции классификации на простом стимульном материале.

Мыслительная деятельность при решении математических задач затруднена уже на самых ранних её этапах, как во время предварительной ориентировки в условиях задачи, так и на стадии формирования стратегии решения задачи. Самостоятельное решение математических задач недоступно, наводящие вопросы специалиста повышают продуктивность работы незначительно.

В целом полученные результаты комплексного нейропсихологического обследования указывают на интеллектуально-мнестическое снижение, зрительно-пространственные нарушения, зрительную предметную агнозию, вторичную акалькулию по префронтальному типу, пространственноконструктивную апраксию, модальнонеспецифические нейродинамические нарушения.

Когнитивные нарушения умеренной степени выраженности. Оценка по итогам выполнения трех шкал: MMSE - 12 баллов, FAB - 11 баллов, госпитальная шкала тревоги и депрессии - соответственно 13 и 7 баллов.

Топический диагноз: в соответствии с результатами анализа данных нейропсихологического обследования констатировано патологическое функционирование лобных (префронтальных и медиобазальных) отделов обоих полушарий головного мозга с вовлечением в патологический процесс теменно-затылочных структур, заинтересованности в процессе гипоталамо-гипофизарных и диэнцефально-стволовых структур головного мозга.

\section{ОБСУЖДЕНИЕ}

В 2011 г. Национальным Институтом по проблемам старения и Альцгеймеровской Ассоциацией (NIA-AA) были предложены новые критерии клинической диагностики БА. Были выделены три стадии развития БА: доклиническая, умеренный когнитивный дефицит, стадия деменции $[10,11,12]$.

\section{Доклиническая стадия БА}

Основой для определения доклинической стадии БА является выявление биомаркеров при нейровизуализационных и лабораторных исследованиях (к ним относятся бета-амилоид, тау-протеин, фосфорилированный тау-протеин) и оценка нейродегенеративных изменений, в частности оценка уровня когнитивного дефицита. Около 40 лет назад B. Reisberg et al. (1982) выдвинули теорию о том, что субъективное когнитивное снижение (СКС) предшествует умеренному когнитивному снижению и является преддементной стадией БА. Для его характеристики использовались различные термины, такие как субъективные когнитивные жалобы, субъективные жалобы на память, субъективные проблемы с памятью, субъективное нарушение памяти, субъективные когнитивные нарушения, потеря субъективной памяти, ухудшение субъективной памяти и субъективный когнитивный спад. Вместе с тем оставалась неопределенность о том, какие жалобы пациента наиболее полезны для выявления очень раннего когнитивного снижения [13].

F. Jessen et al. (2014) не только предложили критерии для диагностики субъективных когнитивных нарушений (табл. 1), но и определили особенности, повышающие вероятность доклинической стадии БА у пациентов с умеренными когнитивными нарушениями [14]. Следует понимать, что прогностическая сила СКС наиболее высока на поздней доклинической стадии БА, когда способность обнаруживать объективные когнитивные нарушения недостаточна из-за ограниченной чувствительности стандартных нейропсихологических тестов [15].

По данным метаанализа 28 исследований, проведенного A.J. Mitchell с коллегами, был сделан вывод, что у пожилых людей с субъективными когнитивными жалобами, без объективного подтверждения когнитивного снижения, вероятность развития деменции в 2 раза выше, чем у лиц без субъективных жалоб. Согласно данным исследователей, субъективные когнитивные жалобы пожилых людей от 2,3\% до 6,6\% случаев будут прогрессировать до умеренного когнитивного расстройства и деменции в течение года [16].

Обзор литературы R. Buckley et al. (2015), выполненный по теме субъективного когнитивного снижения, включает различные модели опыта клинически нормальных пожилых людей с жалобами на снижение когнитивных функций по срав- 
нению с пациентами с умеренным когнитивным расстройством и деменцией при БА [17]. Т а б л и ц а 1. Критерии для диагностики субъективного когнитивного снижения (Jessen F. et al., 2014)

\begin{tabular}{|c|c|}
\hline \multicolumn{2}{|c|}{ Диагностические критерии для выявления СКС } \\
\hline Подтверждающие & Исключающие \\
\hline $\begin{array}{l}\text { Постоянное снижение когнитивных функций, испытан- } \\
\text { ное на собственном опыте, по сравнению с ранее нор- } \\
\text { мальным состоянием и не связанное с острым событием. } \\
\text { Нормальные результаты с учетом факторов возраста, по- } \\
\text { ла и образования в стандартизированных когнитивных } \\
\text { тестах, которые используются для подтверждения уме- } \\
\text { ренного когнитивного расстройства }\end{array}$ & $\begin{array}{l}\text { Легкие когнитивные нарушения, продромальная } \\
\text { стадия БА или деменции. } \\
\text { Состояние может быть объяснено наличием пси- } \\
\text { хического расстройства или неврологического за- } \\
\text { болевания (кроме БА), приемом лекарственных } \\
\text { средств или употреблением психоактивных ве- } \\
\text { ществ }\end{array}$ \\
\hline \multicolumn{2}{|c|}{$\begin{array}{c}\text { Особенности, повышающие вероятность доклинической стадии БА, } \\
\text { у пациентов субъективными когнитивными нарушениями [12] }\end{array}$} \\
\hline \multicolumn{2}{|c|}{$\begin{array}{l}\text { Субъективное когнитивное снижение в течение последних } 5 \text { лет } \\
\text { Возраст появления СКС } 60 \text { лет и выше } \\
\text { Опасения (беспокойства), связанные с СКС } \\
\text { Чувство реализации ниже, чем у других лиц той же возрастной группы } \\
\text { Подтверждение когнитивного снижения исследователем } \\
\text { Наличие АРОЕ } 4 \text { аллеля } \\
\text { Наличие биомаркеров БА (определяющие доклиническую стадию БА) }\end{array}$} \\
\hline
\end{tabular}

Что касается жалоб, то клинически здоровые пожилые люди, как правило, упоминали среди них проблемы с памятью, включая трудности с подбором/поиском слов и имен. Снижение когнитивных способностей часто трактовалось как сопутствующее, причем причинно-следственные связи были связаны со стрессом, тревогой или возрастным фактором. Кроме того, клинически нормальные пожилые люди часто сообщали о гневе, разочаровании или раздражении из-за предполагаемых провалов памяти, что отражает сохраняемую проницательность и осознанное понимание когнитивных проблем [17].

R.F. Buckley et al. (2015) обнаружили, что субъективное когнитивное снижение коррелирует с более выраженной депрессивной симптоматикой и меньшим объемом левого гиппокампа [18].

Таким образом, в настоящее время не существует единого мнения специалистов о том, как следует оценивать или классифицировать субъективные когнитивные нарушения, а также какие использовать при этом подходы, применение различных методов демонстрирует огромную неоднородность позиций. Подтверждением этого является то, что у субъектов с деменцией и умеренным когнитивным расстройством при БА были выявлены более высокие уровни кортизола в спинно-мозговой жидкости, чем у здоровых людей, что может оказывать нейротоксическое действие на гиппокамп и способствовать нарастанию окислительного стресса и токсичности амилоидного $\beta$-пептида $[19,20]$.

Стадия умеренного когнитивного дефицита

Ввиду необходимости правильной диагностики заболевания исследователями были предложе- ны клинические критерии, выработанные на основе жалоб и основных проявлений $[21,22]$.

К ним относятся: 1) беспокойство по поводу изменения познавательной деятельности, проявляющееся в жалобах самого пациента или его близких; 2) нарушения в одной или нескольких когнитивных областях, включая память, исполнительные функции, внимание, язык и зрительнопространственные навыки, не соответствующие возрасту и уровню образования пациента; 3) сохранность функций самообслуживания; 4) когнитивные изменения без признаков значительного ухудшения социального или профессионального функционирования.

Отмечается, что несмотря на то что пациенты испытывают некоторые затруднения при выполнении сложных задач (например, такие как оплата счетов, приготовление пищи, покупка продуктов питания и пр.), тем не менее они сохраняют свою независимость и самостоятельность в повседневной жизни (самообслуживание) при минимальной потребности в поддержке со стороны. Более подробную информацию о их повседневной бытовой деятельности предоставляют родственники. На этапе умеренного когнитивного дефицита для клинициста главной задачей является распознавание этиологии когнитивного снижения, т.е. пока не произошел переход в стадию деменции [23].

В настоящий момент возможно генетическое исследование с целью поиска аутосомнодоминантных мутаций, характерных для БА (мутации в APP, PS1, PS2). В случае если данные мутации выявлены, то с высокой вероятностью когнитивный дефицит является продромальным периодом болезни. Хотя, как отмечают исследо- 
ватели, вопрос о времени перехода из стадии умеренного когнитивного дефицита в стадию деменции, даже при наличии данных мутаций, всё ещё остается без ответа [21].

\section{Стадия деменции}

Следующей стадией развития БА является деменция. Отечественные исследователи выделяют наиболее характерные когнитивные и поведенческие (нейропсихиатрические) симптомы [24]: 1) нарушается способность функционировать на работе или осуществлять обычные виды деятельности; 2) снижение ресурсных возможностей по сравнению с предыдущим уровнем функционирования; 3) происходящее снижение невозможно объяснить делирием или серьезными психическими расстройствами; 4) когнитивные изменения подтверждены самим пациентом, ухаживающим лицом и нейропсихологическим тестированием; 5) когнитивные или поведенческие нарушения затрагивают не менее двух из базовых социальных сфер жизни: память, мышление, суждение, планирование деятельности, нарушение языковых функций (говорение, чтение, письмо), изменение личностного потенциала, нарушение зрительнопространственного мышления (неспособность распознавать знакомые лица или предметы или находить объекты, располагающиеся в прямой видимости, несмотря на хорошую остроту зрения), утрата способности работать с простым ручным инструментом, полная/частичная навыков самообслуживания и передвижения.

При этом ключевым отличием деменции от умеренного когнитивного расстройства остается значительное вмешательство в профессиональнопроизводственное функционирование и выполнение обычных повседневных дел. К дегенеративным изменениям вследствие дементного процесса относятся когнитивные и эмоционально-волевые нарушения [25], обусловливающие снижение социальных навыков человека, в частности способности к социальному продуктивному взаимодействию. Не случайно при прогрессировании любого типа деменции на определенном этапе присоединяется и постепенно нарастает социальная дезадаптация в социально-психологическом статусе: нарушается коммуникативное взаимодействие, ограничиваются повседневная активность и навыки самообслуживания. Как правило, снижение когнитивных функций субъективно не воспринимается самим больным как «психический дефицит», в силу чего начальные признаки развивающегося заболевания раньше замечаются близкими, которые с прогрессированием деменции принимают на себя роль ухаживающего лица.

\section{ЗАКЛЮЧЕНИЕ}

Таким образом, нейропсихологическое исследование психических функций и поведения паци- ентов с расстройством памяти с позиции системного биопсихосоциального подхода не только детализирует когнитивный дефицит, но и отражает степень утраты социальных функций.

\section{КОНФЛИКТ ИНТЕРЕСОВ}

Авторы заявляют об отсутствии явных и потенциальных конфликтов интересов в связи с публикацией данной статьи.

\section{ИСТОЧНИК ФИНАНСИРОВАНИЯ}

Исследование не имело спонсорской поддержки. Авторы несут полную ответственность за предоставление окончательной версии рукописи в печать. Все авторы принимали участие в разработке концепции статьи и написании рукописи.

\section{СООТВЕТСТВИЕ ПРИНЦИПАМ ЭТИКИ}

Исследование было одобрено ЛЭК НМИЦ ПН им. В.М. Бехтерева. Перед началом обследования пациентка подписала форму информированного согласия (заседание ЛЭК от 18.02.2021).

ЛИТЕРАТУРА/REFERENCES

1. Atri A. The Alzheimer's Disease Clinical Spectrum: Diagnosis and Management. Med Clin North Am. 2019 Mar;103(2):263-293. doi: 10.1016/j.mcna.2018.10.009. PMID: 30704681.

2. Weller J, Budson A. Current understanding of Alzheimer's disease diagnosis and treatment. F1000Res. 2018 Jul 31;7:F1000 Faculty Rev-1161. doi: 10.12688/f1000research.14506.1. PMID: 30135715; PMCID: PMC6073093.

3. Alzheimer A. Über eine eigenartige Erkrankung der Hirnrinde. Allgemeine Zeitschrift für Psychiatrie und Psychisch-Gerichtliche Medizin. 1907;64:146148.

4. Alzheimer A. Über eigenartige Krankheitsfälle des späteren Alters. Zeitschrift für die Gesamte Neurologie und Psychiatrie. 1911;4:356-385.

5. Bondi MW, Edmonds EC, Salmon DP. Alzheimer's Disease: Past, Present, and Future. J Int Neuropsychol Soc. 2017 Oct;23(9-10):818-831. doi: 10.1017/S135561771700100X. PMID: 29198280; PMCID: PMC5830188.

6. Blessed G, Tomlinson BE, Roth M. The association between quantitative measures of dementia and of senile change in the cerebral grey matter of elderly subjects. Br J Psychiatry. 1968 Jul;114(512):797811. doi: 10.1192/bjp.114.512.797. PMID: 5662937.

7. Katzman R. Editorial: The prevalence and malignancy of Alzheimer disease. A major killer. Arch Neurol. $\quad 1976$ Apr;33(4):217-8. doi: 10.1001/archneur.1976.00500040001001. PMID: 1259639.

8. Albert ML, Feldman RG, Willis AL. The 'subcortical dementia' of progressive supranuclear palsy. J Neurol Neurosurg Psychiatry. 1974 Feb;37(2):12130. doi: 10.1136/jnnp.37.2.121. PMID: 4819905; PMCID: PMC494589.

9. Bondi MW, Edmonds EC, Salmon DP. Alzheimer's Disease: Past, Present, and Future. J Int Neuropsy- 
chol Soc. 2017 Oct;23(9-10):818-831. doi: 10.1017/S135561771700100X. PMID: 29198280; PMCID: PMC5830188.

10. Jack CR Jr, Albert MS, Knopman DS, McKhann GM, Sperling RA, Carrillo MC, Thies B, Phelps $\mathrm{CH}$. Introduction to the recommendations from the National Institute on Aging-Alzheimer's Association workgroups on diagnostic guidelines for Alzheimer's disease. Alzheimers Dement. 2011 May;7(3):257-62. doi: 10.1016/j.jalz.2011.03.004. Epub 2011 Apr 21. PMID: 21514247; PMCID: PMC3096735.

11. Емелин А.Ю. Новые критерии диагностики болезни Альцгеймера. Неврология, нейропсихиатрия, психосоматика. 2011. Т. 3 (4). С. 5-8. Emelin AYu. New criteria for diagnosing Alzheimer's disease. Neurology, Neuropsychiatry, Psychosomatics. 2011;3(4):5-8 (in Russian). https://doi.org/10.14412/2074-2711-2011-337

12. Коберская Н.Н. Болезнь Альцгеймера. Неврология, нейропсихиатрия, психосоматика. 2019. Т. 11, № 35. C. 52-60. Koberskaya N.N. Alzheimer's disease. Neurology, Neuropsychiatry, Psychosomatics. 2019;11(35):52-60 (in Russian). https://doi.org/10.14412/2074-2711-2019-35-52-60

13. Jessen F. Subjective and objective cognitive decline at the pre-dementia stage of Alzheimer's disease. Eur Arch Psychiatry Clin Neurosci. 2014 Nov;264 Suppl 1:S3-7. doi: 10.1007/s00406-014-0539-z. Epub 2014 Sep 20. PMID: 25238934.

14. Jessen F, Amariglio RE, van Boxtel M, Breteler M, Ceccaldi M, Chételat G, Dubois B, Dufouil C, Ellis KA, van der Flier WM, Glodzik L, van Harten AC, de Leon MJ, McHugh P, Mielke MM, Molinuevo JL, Mosconi L, Osorio RS, Perrotin A, Petersen RC, Rabin LA, Rami L, Reisberg B, Rentz DM, Sachdev PS, de la Sayette V, Saykin AJ, Scheltens P, Shulman MB, Slavin MJ, Sperling RA, Stewart R, Uspenskaya O, Vellas B, Visser PJ, Wagner M; Subjective Cognitive Decline Initiative (SCD-I) Working Group. A conceptual framework for research on subjective cognitive decline in preclinical Alzheimer's disease. Alzheimers Dement. 2014 Nov;10(6):844-52. doi: 10.1016/j.jalz.2014.01.001. Epub 2014 May 3. PMID: 24798886; PMCID: PMC4317324.

15. Rabin LA, Smart CM, Amariglio RE. Subjective Cognitive Decline in Preclinical Alzheimer's Disease. Annual Review of Clinical Psychology. 2017;13(1):369-396. doi:10.1146/annurev-clinpsy032816-045136.

16. Mitchell AJ, Beaumont H, Ferguson D, Yadegarfar M, Stubbs B. Risk of dementia and mild cognitive impairment in older people with subjective memory complaints: meta-analysis. Acta Psychiatr Scand. 2014 Dec;130(6):439-51. doi: 10.1111/acps. 12336. Epub 2014 Sep 13. PMID: 25219393.

17. Buckley RF, Ellis KA, Ames D, Rowe CC, Lautenschlager NT, Maruff P, Villemagne VL, Macaulay SL, Szoeke C, Martins RN, Masters CL, Savage G,
Rainey-Smith SR, Rembach A, Saling MM; Australian Imaging Biomarkers and Lifestyle Study of Ageing (AIBL) Research Group. Phenomenological characterization of memory complaints in preclinical and prodromal Alzheimer's disease. Neuropsychology. 2015 Jul;29(4):571-81. doi: 10.1037/neu0000156. Epub 2015 Feb 9. PMID: 25664464.

18. Buckley RF, Maruff P, Ames D, Bourgeat P, Martins RN, Masters CL, Rainey-Smith S, Lautenschlager N, Rowe CC, Savage G, Villemagne VL, Ellis KA; AIBL study. Subjective memory decline predicts greater rates of clinical progression in preclinical Alzheimer's disease. Alzheimers Dement. 2016 Jul;12(7):796-804. doi: 10.1016/j.jalz.2015.12.013. Epub 2016 Feb 4. PMID: 26852195.

19. Donohue MC, Sperling RA, Salmon DP, Rentz DM, Raman R, Thomas RG, Weiner M, Aisen PS; Australian Imaging, Biomarkers, and Lifestyle Flagship Study of Ageing; Alzheimer's Disease Neuroimaging Initiative; Alzheimer's Disease Cooperative Study. The preclinical Alzheimer cognitive composite: measuring amyloid-related decline. JAMA Neurol. 2014 Aug;71(8):961-70. doi: 10.1001/jamaneurol.2014.803. PMID: 24886908; PMCID: PMC4439182.

20. Curto M, Martocchia A, Ferracuti S, Comite F, Scaccianoce S, Girardi P, Nicoletti F, Falaschi P. Increased Total Urinary Cortisol (tUC) and Serum Brain-derived Neurotrophic Factor (BDNF) Ratio in Alzheimer Disease (AD)-affected Patients. Alzheimer Dis Assoc Disord. 2017 Apr-Jun;31(2):173176. doi: 10.1097/WAD.0000000000000156. PMID: 27196536.

21. Albert MS, DeKosky ST, Dickson D, Dubois B, Feldman HH, Fox NC, Gamst A, Holtzman DM, Jagust WJ, Petersen RC, Snyder PJ, Carrillo MC, Thies B, Phelps CH. The diagnosis of mild cognitive impairment due to Alzheimer's disease: recommendations from the National Institute on Aging-Alzheimer's Association workgroups on diagnostic guidelines for Alzheimer's disease. Alzheimers Dement. 2011 May;7(3):270-9. doi: 10.1016/j.jalz.2011.03.008. Epub 2011 Apr 21. PMID: 21514249; PMCID: PMC3312027.

22. Пилипович А.А., Воробьева О.В. Умеренные когнитивные расстройства: современные аспекты диагностики и терапии. Журнал неврологии и психиатрии им. С.С. Корсакова. 2020. Т. 120, № 11. C. 124-130. Pilipovich AA, Vorobieva OV. Mild cognitive impairment: modern aspects of diagnostics and therapy. S.S. Korsakov Journal of Neurology and Psychiatry. 2020;120(11):124-130 (in Russian). https://doi.org/10.17116/jnevro2020120111124

23. Емелин А.Ю., Литвиненко И.В., Лобзин В.Ю. Ошибки в ведении пациентов с болезнью Альцгеймера: анализ проблем и пути их решения. Неврология, нейропсихиатрия, психосоматика. 2019. T. 11, № 4. C. 141-146. Emelin AYu, 
Litvinenko IV, Lobzin VYu. Errors in the management of patients with Alzheimer's disease: analysis of problems and solutions. Neurology, Neuropsychiatry, Psychosomatics. 2019;11(4):141-146 (in Russian). https://doi.org/10.14412/2074-27112019-4-141-146

24. Науменко А.А., Громова Д.О., Трофимова Н.В., Преображенская И.А. Диагностика и лечение болезни Альцгеймера. Неврология, нейропсихиатрия, психосоматика. 2016. Т. 8, № 4. С. 91-97. Naumenko AA, Gromova DO, Trofimova NV, Preobrazhenskaya IA. Diagnosis and treatment of Alzheimer's disease. Neurology, Neuropsychiatry, Psychosomatics. 2016;8(4):91-97 (in Russian). https://doi.org/10.14412/2074-2711-2016-4-91-97
25. Боженкова К А., Бохан Т.Г., Терехина О.В. Особенности субъективного качества жизни у лиц пожилого возраста с опытом переживания геронтологического насилия. Вестник Кемеровского государственного университета. 2018. № 1. C. 90-101. Bozhenkova KA, Bokhan TG, Terekhina OV. Subjective Quality of Life of Senior Citizens who Experienced Gerontological Abuse. Bulletin of Kemerovo State University. 2018;1:90101 (in Russian). DOI:10.21603/2078-8975-2018$1-90-101$

Поступила в редакцию 1.03.2021 Утверждена к печати 28.05.2021

Коцюбинская Юлия Вадимовна, к.м.н., ведущий научный сотрудник отделения реабилитации психоневрологических больных. ORCID iD 0000-0001-9881-5942. ResearcherID Q-6749-2016. AuthorID 701200.

Казаков Антон Валерьевич, аспирант отделения реабилитации психоневрологических больных. ORCID iD 0000-0001-5320-1541. antonik854@mail.ru

Матюшкина Анна Александровна, младший научный сотрудник, нейропсихолог, медицинский психолог отделения медицинской психологии и психодиагностики. SPIN-код 4650-0240. ResearcherID E-3545-2014, AuthorID 910773. ORCID iD 0000-0001-6821-7773. anna.matyushkina@ bekhterev.ru

Орлов Илья Аркадьевич, научный сотрудник отделения нейрохирургии. ORCID iD 0000-0001-8418-2144. cipor@inbox.ru

Коцюбинская Юлия Вадимовна, platonk-juliak@yandex.ru 
For citation: Kotsyubinskaya Yu.V., Kazakov A.V., Matyushkina A.A., Orlov I.A. Opportunities of applying neuropsychological research methods in Alzheimer's disease (in terms of a clinical case). Siberian Herald of Psychiatry and Addiction Psychiatry. 2021; 2 (111): 64-72. https://doi.org/10.26617/1810-3111-2021-2(111)-64-72

\title{
Opportunities of applying neuropsychological research methods in Alzheimer's disease (in terms of a clinical case)
}

\author{
Kotsyubinskaya Yu.V., Kazakov A.V., Matyushkina A.A., Orlov I.A.
}

Federal State Budgetary Institution "V.M. Bekhterev National Medical Research Center for Psychiatry and Neurology" of Ministry of Health of the Russian Federation

Bekhterev Street 3, 192019, St. Petersburg, Russian Federation

\begin{abstract}
Alzheimer's disease (AD) is the most common cause of cognitive deficits due to loss of synaptic connections in people over 65 , reaching $80 \%$ of cases of dementia. Despite the rich and productive history of AD study, there are many gaps, especially in the plane of the clinical features of AD course. Objective: a clinical review conducted from the modern standpoint of evidence-based psychiatric practice, based on the observation of the progressive course of cognitive decline in AD disease. A clinical example of a neuropsychological study of a patient with probabilistic AD (diagnosis according to ICD10: F00.1 Dementia in AD with late onset G30.1+) with interpretation of the obtained data is presented. The patient underwent therapy at the Center for the Treatment of Memory Disorders of the FSBI V.M. Bekhterev National Medical Research Center for Psychiatry and Neurology of Ministry of Health of the Russian Federation (St. Petersburg). The results of a comprehensive neuropsychological examination of the patient indicate intellectual-mnestic decline, visual-spatial disturbances, visual object agnosia, secondary acalculia of the prefrontal type, spatial-constructive apraxia, modal-nonspecific neurodynamic disorders. Cognitive impairment of moderate severity was revealed. According to the results of the assessment on three scales, the following points were obtained: The Mini-Mental State Examination (MMSE) - 12 points, the Frontal Assessment Battery (FAB) - 11 points, the Hospital Anxiety and Depression Scale (HADS) - 13 points and 7 points. Topical diagnosis of brain damage: the results of neuropsychological examination indicate pathological functioning of the frontal (prefrontal and mediobasal) parts of both hemispheres of the brain with the involvement of the parieto-occipital structures in the pathological process, interest in the process of the hypothalamic-pituitary and diencephalic-brainstem structures of the brain. Thus, a neuropsychological study of mental functions in patients with memory disorders from the standpoint of a biopsychosocial approach details not only cognitive deficits, but also the degree of loss of social functions.
\end{abstract}

Keywords: Alzheimer's disease, cognitive impairment, mild cognitive deficit, dementia, neuropsychological methods, analysis of mental functions.

Kotsyubinskaya Yulia V., PhD, lead researcher, Department of Rehabilitation of Neuropsychiatric Patients, V.M. Bekhterev National Medical Research Center for Psychiatry and Neurology, St. Petersburg, Russian Federation. ORCID iD 0000-0001-9881-5942. ResearcherID Q-6749-2016. AuthorID 701200.

Kazakov Anton V., postgraduate student, Department of Rehabilitation of Neuropsychiatric Patients, V.M. Bekhterev National Medical Research Center for Psychiatry and Neurology, St. Petersburg, Russian Federation. ORCID iD 00000001-5320-1541. antonik854@mail.ru

Matyushkina Anna A., junior researcher, neuropsychologist, medical psychologist, Department of Medical Psychology and Psychodiagnostics, V.M. Bekhterev National Medical Research Center for Psychiatry and Neurology, St. Petersburg, Russian Federation. SPIN-code 4650-0240, ResearcherID E-3545-2014, AuthorID 910773, ORCID iD 00000001-6821-7773. anna.matyushkina@bekhterev.ru

Orlov Ilya A., researcher, Department of Neurosurgery, V.M. Bekhterev National Medical Research Center for Psychiatry and Neurology, St. Petersburg, Russian Federation. ORCID iD 0000-0001-8418-2144. cipor@inbox.ru

Kotsyubinskaya Yulia V., platonk-juliak@yandex.ru 